\title{
Validation of Vortex-Lattice Method for Loads on Wings in Lift-Generated Wakes
}

\author{
Vernon J. Rossow* \\ NASA Ames Research Center, Moffett Field, California 94035-1000
}

\begin{abstract}
A study is described that evaluates the accuracy of vortex-lattice methods when they are used to compute the loads induced on aircraft as they encounter lift-generated wakes. The evaluation is accomplished by the use of measurements made in the 80 by $120 \mathrm{ft}$ Wind Tunnel of the lift, rolling moment, and downwash in the wake of three configurations of a model of a subsonic transport aircraft. The downwash measurements are used as input for a vortex-lattice code in order to compute the lift and rolling moment induced on wings that have a span of $0.186,0.510$, or 1.022 times the span of the wake-generating model. Comparison of the computed results with the measured lift and rolling-moment distributions the vortex-lattice method is very reliable as long as the span of the encountering or following wing is less than about 0.2 of the generator span. As the span of the following wing increases above 0.2 , the vortex-lattice method continues to correctly predict the trends and nature of the induced loads, but it overpredicts the magnitude of the loads by increasing amounts.
\end{abstract}

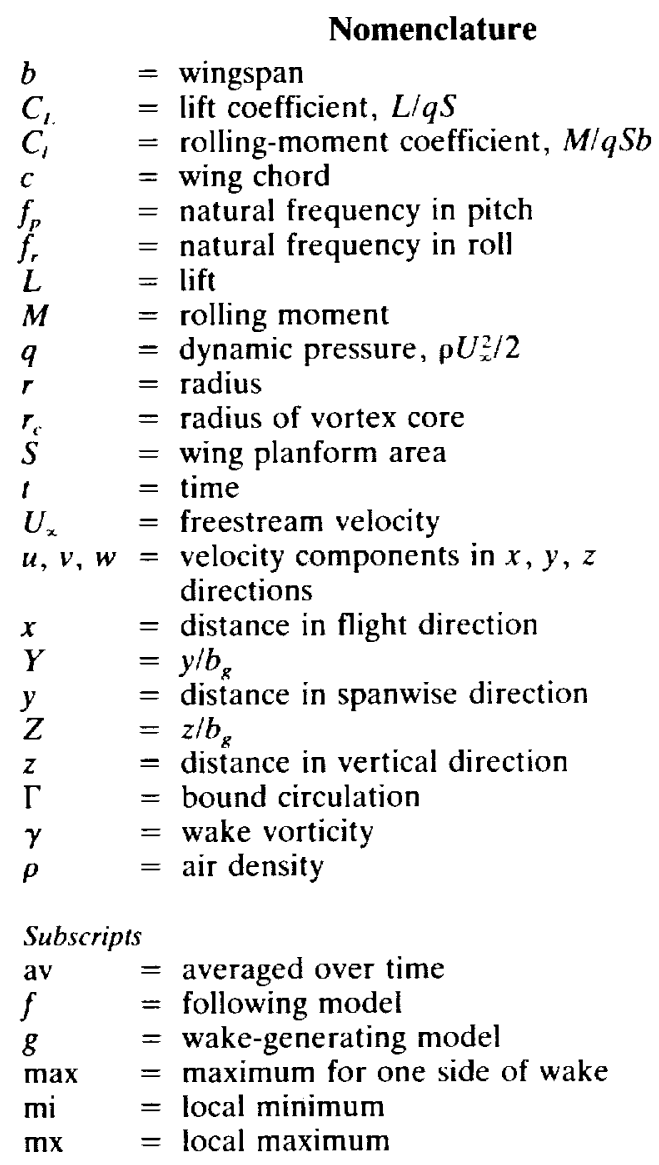

Received April 5, 1994; presented as Paper 94-1839 at the AIAA 12th Applied Aerodynamics Conference, Colorado Springs, CO, June 20-23, 1994; revision received March 23, 1995; accepted for publication May 15, 1995. Copyright (C) 1995 by the American Institute of Aeronautics and Astronautics, Inc. No copyright is asserted in the United States under Title 17, U.S. Code. The U.S. Government has a royalty-free license to exercise all rights under the copyright claimed herein for Governmental purposes. All other rights are reserved by the copyright owner.

${ }^{*}$ Senior Scientist. Associate Fellow AIAA.

\section{Introduction}

7 HROUGHOUT the NASA Federal Aviation Administration (FAA) wake-vortex research program, effort has been directed at the development of reliable models and tools for the representation and prediction of the structure and dynamics of vortex wakes and their interaction with aircraft. As a part of this effort, the study reported here was carried out in order to obtain an improved understanding of the current capability to calculate loads on lifting surfaces embedded in the rotary flowfields of vortex wakes. It was reasoned that the method to be studied must be easy to apply to a large variety of configurations because the number of aircraft configurations in the subsonic transport fleet is large. Another reason for simplicity is that the method must not use much computer time (i.e., it should be fast), so that a large number of solutions can be obtained quickly for spatial analyses and for simulation of the dynamics of aircraft-wake encounters. Along with the foregoing requirements, the method should also be accurate enough so that, given a reliable velocity field as input, the computed lift and rolling moment fall within the scatter of the values measured in the wind tunnel.

A method that possibly fulfills these requirements is the same vortex-lattice method used in previous wake-vortex studies. ${ }^{1.2}$ A validation of the method requires that up- and downwash velocity distributions are available in the same locations in the vortex wake as are the measurements of the lift and rolling moment on following wings of various sizes. In order to reduce the uncertainties in the comparisons to be made, both the measured velocity field and the measured lift and rolling moment are taken from the same wind-tunnel test. ${ }^{3}$ With the measured velocity distribution in the wake used as a boundary condition, the vortex-lattice code is used to compute lift and rolling moment on the following wing. The computed values are then compared with the measured values to assess the validity of the method. In this way the vortex-lattice method to be tested is the only theoretical link in the comparisons to be made. The comparisons to be discussed also provide an evaluation of the experimental techniques used in the experiment.

A variety of theoretical methods could have been tested as the link between the computed and measured lift and rolling moments. Only the vortex-lattice method is used in the comparisons because it is simple to apply and has the capability to include a variety of wing shapes and vortex structures. 
SMSA/TM-95- 207301

NLS
NO2- TM

Validation of Vortex-Lattice Method for Loads on Wings in Lift-Generated Wakes Vernon J. Rossow

Reprinted from

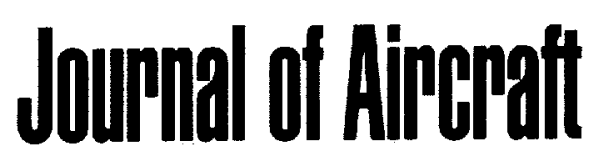

Volume 32, Number 6, Pages 1254-1262

\section{OAIAA.}

A publication of the

American Institute of Aeronautics and Astronautics, Inc.

370 L'Enfant Promenade, SW

Washington, DC 20024-2518 
Therefore, all approximate methods such as those based on strip theory, ${ }^{2}$ and those based on global-type arguments, ${ }^{4.5}$ were discarded as too unreliable for the comparisons being made, even though they can provide insight into vortex/wing interactions. Also, it is reasoned that the oncoming stream may not be considered as a shear flow composed of a spanwise distribution of vertical velocity. Not only is the shear flow solenoidal and not potential, but the vertical momentum in the vertical shear layers is infinite rather than finite, as in a vortex.

This article first reviews some of the assumptions that need to be made in order to apply the vortex-lattice method to vortex wakes. The experimental setup and procedures used to obtain the velocity and loads data are then described along with the methods used to adjust the data for model misalignments and for vortex meander. Computations are then presented to evaluate the accuracy of the vortex-lattice method.

\section{Applicability of Vortex-Lattice Method}

The vortex-lattice method chosen for this study is based on potential flow theory that assumes that the flowfield is steady with time, incompressible, inviscid, and that flow separation or stall is negligible. Since the velocity field that interacts with the following or encountering wing is brought about by a vortex wake, the flowfield is solenoidal and not potential. Therefore, if a potential-flow method like the vortex-lattice method is to be used in lift-generated wakes, it must either be modified in some way or the flowfield must be reinterpreted. If the velocity field is impressed by a vortex system the boundary conditions on the various lattices that represent the wing may be specified by 1) assuming that each panel needs to respond to the local flow angle or 2) that the wing panels are inclined at an angle to a uniform freestream that is equal to the angle of attack impressed by the vortex system. In either case, the strengths of the vortex-lattice panels are adjusted so that there is no flow through the encountering wing at the control points.

In the vortex-lattice method the panels consist of horseshoe vortices. The cross-stream component of the horseshoe is swept or unswept in order to best fit the planform of the wing while the other two legs of the horseshoe extend in the downstream direction to infinity. All three legs of the horseshoe lie in a $z=$ constant plane, which is where the wing camber and the up- and downwash distribution is applied; i.e., a thin-wing boundary condition. The method employs only the one layer of lattices and does not include a separate lattice or panel system to represent the upper and lower surfaces of the wing or tail surfaces being considered. When the geometry of the horseshoe lattice has been established, the strengths of the horseshoe vortices are set in combination by a matrix inversion so that the boundary conditions on the wing are satisfied.

When such a potential flow technique is applied to a wing placed in a rotating and rotational (or solenoidal) flowfield, the applicability of the predictions is, at least academically, open to question. That is, since the flowfield of the vortices is rotational and not potential, the use of a potential-flow method is, in principal, not valid. If, however, it is assumed that the flowfield and wing are reconfigured so that the freestream is uniform rather than rotational, and that the wing is twisted to match the angle of attack imposed by the vortex, the problem is restored to being potential. This way of treating the overall problem was pointed out when discussions" "called attention to a reciprocal theorem developed by Heaslet and Spreiter, ${ }^{12}$ which relates an arbitrary downwash field to that of fictitious wings in steady rolling motion. In brief, the theorem formalizes and justifies the assumption, which is usually made, that the loading on the encountering wing can be calculated by use of the assumption that the oncoming stream is uniform rather than rotational, and the vortex downwash field is interpreted as wing twist (or as local angle of attack).
To find out how well the vortex-lattice method represents the interaction of a wing with the vortical flowfields of liftgenerated wakes, a potential flow analysis based on Munk's crossflow theory ${ }^{13}$ was carried out for the interaction of a potential vortex filament with a wing of low AR. ${ }^{14}$ When the loads predicted by the vortex-lattice and crossflow methods are compared, it is found that the distributions and the total values of lift and rolling moment are in very good agreement when the encountering wing has an AR less than one. As the $A R$ increased above one, the difference between the results increased because Munk's crossflow theory is based on slender-body theory. In both the crossflow theory and the vortexlattice method, it is assumed that the path of the vortex filament is not altered by the presence of the following wing as it encounters the wake vortices. Therefore, the comparisons made ${ }^{1+}$ indicate that if the structure of the vortical flowfield in the freestream approaching the wing is not significantly distorted by the presence of the encountering wing, the vortex-lattice method is a reliable indicator of wake-vortex induced loads.

\section{Experimental Setup and Test Procedures}

The data obtained during the experimental program are the lift and rolling moment induced on a following wing, ,, 3 and the velocity distribution in the flowfield of the vortex wake as measured with a hot-film anemometer probe. As an aid to the understanding of the adjustments and the use of the data in the comparisons, a brief description of the experiment ${ }^{3}$ is repeated here. Figure 1 presents a diagram of the plan view and Fig. 2 a photograph of the test installation. The wakegenerating model $(0.03$-scale model of the B-747 is mounted on a single strut with a strain-gauge balance to measure its lift, drag, and pitching moment. An 81 -ft test distance (corresponds to a full-scale distance of one-half mile) was used in the experiment so that direct comparisons could be made with results obtained in previous tests..$^{3.15 .16}$ An inverted mounting of the wake-generating model is used to minimize interference of the strut wake with the vortex wake of the generator model (Fig. 2). The model wake then moves upward away from the strut wake that tends to go straight downstream. The angle of attack of the generator is set remotely through an actuator and indicator mechanism.

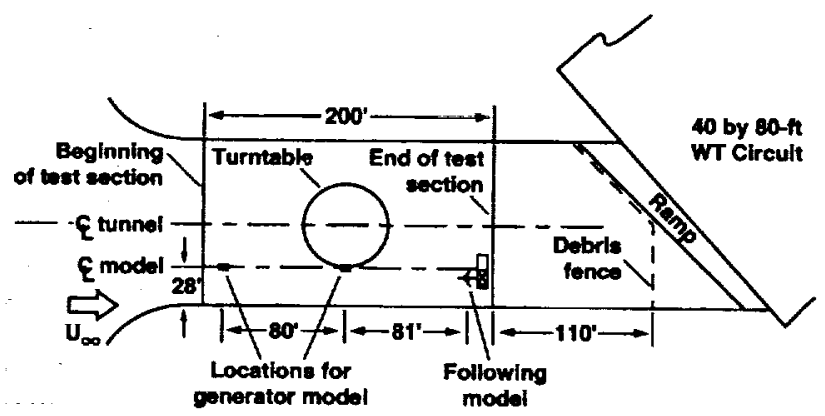

Fig. 1 Plan view of setup of experiment in 80 by $120 \mathrm{ft}$ Wind Tunnel.

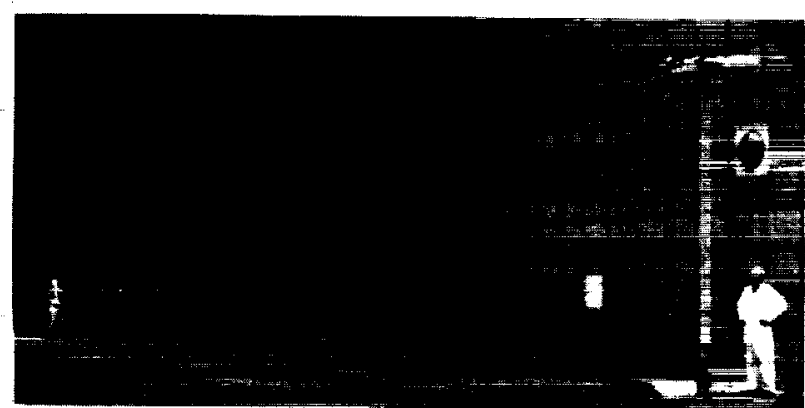

Fig. 2 Photograph of experimental setup in 80 by $120 \mathrm{ft}$ Wind Tunnel. 
Table 1 Wind-tunnel conditions ${ }^{3}$

Freestream velocity $=U_{x}=131 \mathrm{ft} / \mathrm{s}$

Dynamic pressure $=q_{x}=20 \mathrm{lb} / \mathrm{ft}^{2}$

Reynolds number based on $\bar{c}_{g}=R e_{g}=660,000$

Distance to follower model $=x_{f}=81 \mathrm{ft}$

Table 2 Data on wake-generating (B-747) model $^{3}$

Wingspan $=b_{g}=70.5$ in

Average chord of wing $=\bar{c}_{g}=10.1$ in.

$\mathrm{AR}=\mathrm{AR}_{\mathrm{g}}=6.96$

Wing area $=S_{\mathrm{g}}=4.94 \mathrm{ft}^{2}$

Horizontal stabilizer angle $=0 \mathrm{deg}$

Landing gear deployed flaps, deg

$30 / 30$, no fins

$30 / 30,4 \times 6$ in. fins, $\alpha_{\mathrm{fin}}=+18 \mathrm{deg}$

$30 / 0$, no lins

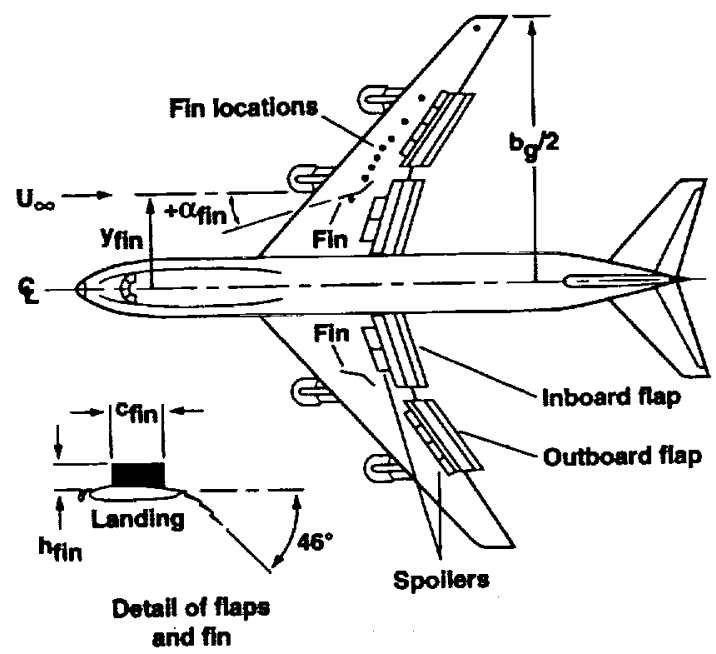

Fig. 3 Diagram of wake-generating model (B-747) that illustrates how wing fins are positioned to alleviate wake velocities.

The wake-generating model is tested with its landing gear, leading-edge slats, and trailing-edge flaps deployed (Table 1). As in the past, and as indicated in Table 2 , a designation of flaps $(30 / 30 \mathrm{deg})$ is used to indicate that the trailing-edge flaps are fully deployed and that the aircraft is in its full-landing configuration with leading-edge slats and landing gear deployed. In the $(30 / 0 \mathrm{deg})$ or modified-landing configuration, the inboard landing flaps are fully deployed and the outboard ones are stowed. The rest of the model is in its landing configuration. A third configuration (Fig. 3 and Table 2) consists of the landing, or $(30 / 30-\mathrm{deg})$ configuration with a fairly large fin mounted on the upper surface of each side of the wing to accelerate the dispersion of the vortex wake. ${ }^{15,16}$ This configuration is designated as (30/30-deg) fins.

The following-wing models (Table 3) are made with a NACA 0012 airfoil section and have the planforms shown in Fig. 4. Each following-wing model to be tested is mounted on a strut (Fig. 2) that can be raised and lowered over a height of about $8 \mathrm{ft}$ by means of a vertical-traverse mechanism. The verticaltraverse mechanism, in turn, is attached to a tower that can be translated laterally, or spanwise, across the airstream over a range of about $18 \mathrm{ft}$. The following-wing or wake-encountering model is attached to its strut through a strain-gauge balance located inside the centerbody of the wing so that the wake-induced lift and rolling moment can be measured. Both a 1 - and 2-in. internal-type balance were used in the tests to more adequately cover the wide range of loads that occur when the smallest to the largest following wings encounter a vortex wake.
The procedure used to make a measurement in the wake of the generator model consists of several steps. The wakegenerating model is first set up in its desired configuration and angle of attack. The forces and moments on the wakegenerating model were found to change little with time so that time-averaged values are used to evaluate the various coefficients. ${ }^{3}$ After the loads on the wake-generating model are recorded, the following wing is placed at a selected downstream station ( $81 \mathrm{ft}$ for the present test) and at a chosen spanwise and vertical position relative to the streamwise centerline of the wake-generating model. The lift and rolling moment induced on the following wing by the vortex wake are then measured.

If the airstream is perfectly uniform and the vortex wake is steady, different locations of the following wing relative to the wake-generating model cause different amounts of lift and rolling moment to be induced on the following wing. Furthermore, the induced loads would not vary with time. In most wind tunnels, however, the induced loads change with time as the wake vortices move about in the wind tunnel in response to the turbulent eddies in the airstream. Since these perturbation velocities have a substantial distance $(81 \mathrm{ft})$, or time, over which to influence the vortex positions, movement or meander of the vortex wake may not be negligible at the station where the following model or wing is located. In the 80 by $120 \mathrm{ft}$ Wind Tunnel where the test was conducted, the vortices appear to meander from their time-averaged location up to about 2 in. $\left(0.0284 b_{g}\right)$ at a distance of $81 \mathrm{ft}\left(13.8 b_{k}\right)$ behind the wake-generating model: Hence, measurements made in or near a vortex core with a small radius may vary considerably with time.

In order to determine the variation of the measured quantities with time and to find the maximum at each location, the time-varying lift, rolling moment, and velocities are recorded. The final or permanent records are obtained by first calculating the value of each parameter when time-averaged over $0.1 \mathrm{~s}$. In order to obtain one full minute of data, 600 such samples are recorded end-to-end. The permanent or retained record consists of the maximum and the minimum of all of the $0.1-\mathrm{s}$ values that occurred during the 1-min test interval, and also the average or mean value of all 600 samples. A total sample of about $1 \mathrm{~min}$ in duration was found to be long enough that two to four peak values of about the same magnitude would occur. The measured results were written on a magnetic disk for later use, along with the position of the centerline of the following wing (or hot-wire probe) relative to the centerline of the wake-generating model

A wake is analyzed experimentally by first taking data with the following-wing model at a number of spanwise and vertical positions until the entire active wake region has been surveyed $^{3}$ is to find the maximum or most intense value of the rolling moment (positive or negative). If the span of the following model is small relative to the span of the generator model, the largest rolling moment is assumed to occur when

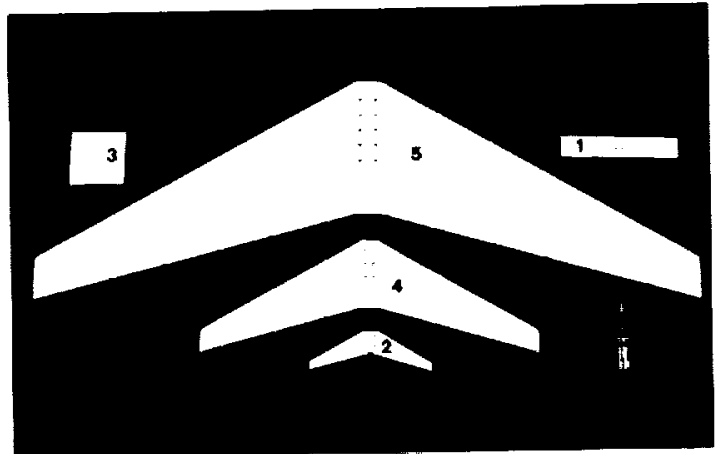

Fig. 4 Overhead photograph of following models used in test program. 
Table 3 Data on following models ${ }^{3}$

\begin{tabular}{|c|c|c|c|c|c|c|c|c|c|c|}
\hline No. & $\begin{array}{l}b_{f} \\
\text { in. }\end{array}$ & $\begin{array}{l}c_{c t}, \\
\text { in. }\end{array}$ & $\begin{array}{l}c_{\text {itip, }} \\
\text { in. }\end{array}$ & $\begin{array}{l}\Lambda_{\text {L.: }} \\
\text { deg }\end{array}$ & $\begin{array}{c}\Lambda_{\mathrm{ret}}, \\
\mathrm{deg}\end{array}$ & $\mathrm{AR}_{r}$ & $\begin{array}{l}S_{f} \\
\mathrm{ft}^{2}\end{array}$ & $b_{j} / b_{k}$ & $\begin{array}{l}f_{,} \\
\mathrm{Hz}\end{array}$ & $\begin{array}{l}f_{1,} \\
\mathrm{~Hz}\end{array}$ \\
\hline 1 & 13.12 & 2.41 & 2.41 & 0 & 0 & 5.44 & 0.220 & 0.186 & 156 & 13.0 \\
\hline 2 & 13.13 & 2.84 & 0.85 & 30 & 15 & 7.14 & 0.168 & 0.186 & 156 & 13.0 \\
\hline 3 & 6.00 & 6.00 & 6.00 & 0 & 0 & 1.00 & 0.250 & 0.085 & 190 & 12.0 \\
\hline 4 & 35.97 & 7.86 & 2.41 & 30 & 15 & 7.00 & 1.283 & 0.510 & 30.5 & 11.5 \\
\hline 5 & 72.04 & 15.67 & 4.72 & 30 & 15 & 7.06 & 5.100 & 1.022 & 12.0 & 7.3 \\
\hline
\end{tabular}

Table 4 Locations of maximum rolling moments and lateral surveys

\begin{tabular}{|c|c|c|c|c|c|c|}
\hline \multirow[b]{2}{*}{ Configuration of generator } & \multirow{2}{*}{$\begin{array}{l}\text { Run } \\
\text { no. }\end{array}$} & \multirow{2}{*}{$\begin{array}{l}\text { Following } \\
\text { wing }\end{array}$} & \multicolumn{2}{|c|}{ Port side } & \multicolumn{2}{|c|}{ Starboard side } \\
\hline & & & $Y_{\operatorname{mix}}$ & $Z_{\text {mix }}$ & $Y_{\max }$ & $Z_{\text {max }}$ \\
\hline \multirow[t]{4}{*}{ Landing, flaps $(30 / 30 \mathrm{deg})$} & 13 & 1 & -0.370 & $-0.471^{: 1}$ & +0.427 & $-0.342^{\prime \prime}$ \\
\hline & $19 \mathrm{~A}$ & 4 & $\overline{-0.342}$ & $-0.471^{\prime \prime}$ & $+\overline{0.370}$ & $-\overline{0.342}$ \\
\hline & 35 & 5 & -0.367 & $-0.471^{\circ}$ & +0.370 & -0.352 \\
\hline & 36 & Hot-film & - & $-0.47 l^{: 1}$ & - & -0.342 \\
\hline \multirow[t]{4}{*}{ Modified landing, flaps $(30 / 0 \mathrm{deg})$} & $15 \mathrm{~A}$ & 1 & -0.475 & $-0.627^{a}$ & +0.501 & -0.426 \\
\hline & 17 & 4 & -0.475 & $-0.627^{a}$ & +0.501 & -0.426 \\
\hline & 34 & 5 & -0.430 & -0.627 & +0.398 & -0.482 \\
\hline & 38 & Hot-film & - & $-0.627^{*}$ & - & $-0.426^{1}$ \\
\hline Landing, flaps $(30 / 30 \mathrm{deg})$ with & 23 & 1 & -0.372 & -0.512 & +0.431 & -0.400 \\
\hline fins, $\alpha_{t \text { tin }}=18 \mathrm{deg}, y_{\mathrm{tin}} / b_{g}=$ & 25 & 3 & $\underline{-}$ & $-0.512^{\prime \prime}$ & $\ldots$ & $\underline{-}$ \\
\hline \multirow[t]{3}{*}{0.480} & 26 & 4 & -0.397 & $-0.512^{\prime \prime}$ & +0.457 & -0.400 \\
\hline & 33 & 5 & -0.313 & -0.512 & +0.340 & -0.454 \\
\hline & 37 & Hot-film & 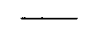 & $-0.512^{i \prime}$ & - & $-0.400^{\mathrm{at}}$ \\
\hline
\end{tabular}

Spanwise survey taken.

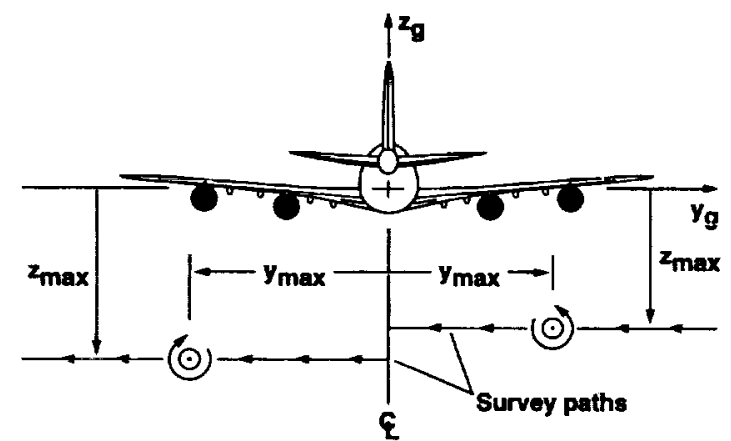

Fig. 5 Illustration of elevation change at wake centerline of path used to carry out spanwise surveys with following models and with hot-film anemometer probe.

the centerline of the following model is aligned with the axis of one of the vortices in the wake. These values are then used as the most hazardous values for the configuration at that distance behind the wake-generating model and as the centers of the vortex locations.

The foregoing procedure that is used to conduct the lateral or spanwise surveys of induced lift and rolling moment with the following-wing/strain gauge balance combination is also used to measure the up- and downwash velocity distributions with a hot-film probe. Now, however, the survey points are all selected at a constant elevation along a spanwise line from the centerline of the wake-generating model out to well beyond the wingtip. In most cases the vortex center on one side of the centerline is different from that on the other side so that the survey line is at a different elevation on each side of the wake (Fig. 5). The centers of the vortices are indicated as $y_{\text {max }}$ and $z_{\text {max }}$ because they are identified as the locations where the rolling moment on a small following wing is a maximum for that side of the centerline.

\section{Measured Flowfield Data}

\section{Overview of Experimental Data}

After the locations were found for the maximum rolling moments imposed by the vortex wake on an encountering

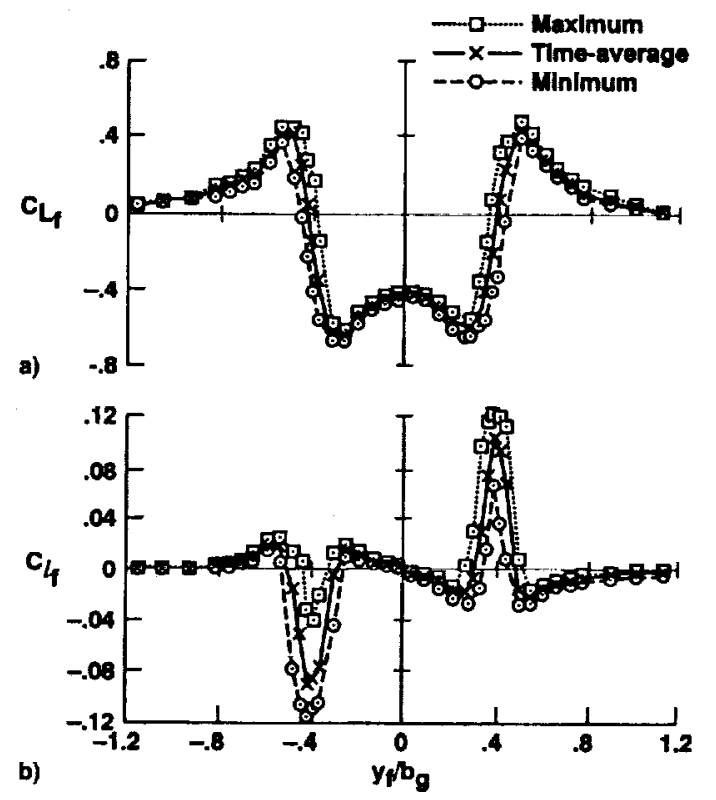

Fig. 6 Typical measured loads on following wing no. 1 as it makes a lateral traverse through wake of (30/30-deg) configuration: a) lift and b) rolling moment.

wing, spanwise surveys were made with following wings nos. 1,4 , and 5 , and with a hot-film anemometer probe. The data in Fig. 6 for following wing no. 1 illustrates the general character of the lift and rolling-moment curves, the number of points taken, and the differences in the maximum, minimum, and time-averaged data. The data taken with a hot-film anemometer probe (Fig. 7) provides the up- and downwash velocity distributions that are used with the vortex-lattice method to compute the spanwise lift and rolling-moment distributions. Table 4 lists the configurations of the wake generator, the various following wings or hot-film anemometer probe for which a survey was taken, and the locations where the most intense rolling moments occurred on each side of the wake centerline. In the hot-film surveys, only streamwise 

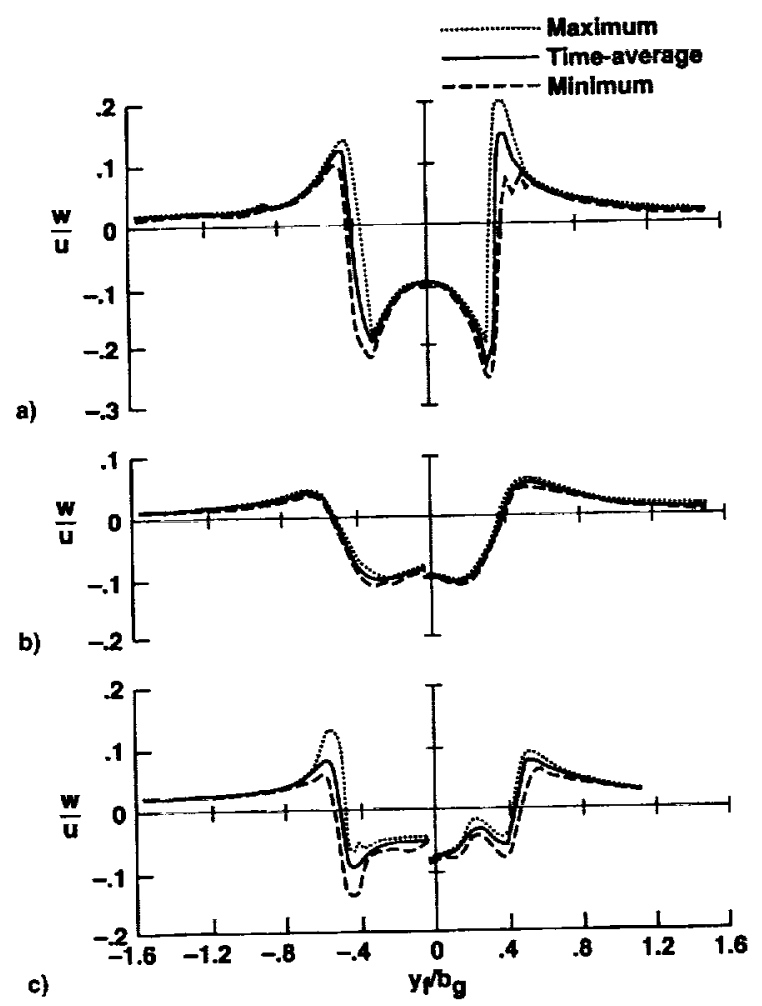

Fig. 7 Up-and downwash distributions measured with hot-film probe along a lateral traverse through vortex centers of wake: a) $30 / 30 \mathrm{deg}$, b) 30/30-deg fins, and c) 30/0 deg.

and vertical velocity components were measured. The two velocity components were then combined to yield the distribution of up- and downwash angle $w / u$ across the wake (Fig. 7).

\section{Adjustments to Experimental Data}

Before the data points were plotted in the various figures or used in the vortex-lattice code, an increment, $\Delta y=0.028 b_{g}$ (i.e., 2 in.), was first applied to the spanwise location to correct for a lateral offset in the measured alignment of the wind-tunnel setup. (A 2 -in. distance in the wind tunnel becomes $\Delta y / b_{g}=2 / 70.5=0.0284$ in dimensionless units.) That is, in all of the spanwise surveys, the data first had to be shifted to the left so that the centerline of the data is aligned with the centerline of the wake-generating model. An upward shift is also needed in the measured upwash angle and lift to compensate for such items as flow angularity in the wind tumnel, any nonzero angle of attack of the following wing or hot-film gauge relative to horizontal, and possible deflections of the support sting, traverse tower, and rail assembly due to air loads from the freestream velocity. Although these displacements are each small, the combination appears to be enough to cause an offset in the measured curves of about $C_{t}=0.02$ and $C_{l l}=0.02$. The offset in the torque distributions is determined so that the curves for rolling moment far from the centerline have about the same magnitude of rolling moment. Such an adjustment is based on the assumption that a slight twist or lateral asymmetry in the following wings causes a bias in all of the data in the distribution.

Next, adjustments were determined for the upwash readings because they are needed to determine the offset of the data from the zero level. The procedure used to find the proper amount of upwash adjustment for the data is based on the assumption that the vortices are very nearly axisymmetric in their core region. It is assumed, therefore, that near the vortex center, the fluid motion is axisymmetric because the swirl velocity dominates both the contributions of the opposite vortex and the perturbation velocities in the free- stream. If this is the case, the maximum upward and downward velocities on each side of the vortex center (i.e., the core velocities) should be equal when the influence of the opposite vortex has been removed. Since the so-called steadystate location of the vortex centers is desired for the adjustments to be made, the time-averaged data distributions are used. An adjustment in vertical velocity that is sufficient to make the up- and downwash equal includes both the correction and the downwash contribution from the opposite vortex. At this point the two cannot be separated. The amount of adjustment required to make the up- and downwash equal on both sides of the vortex center, is the value used as the correction to the data for the zero upwash level.

The downwash distribution of the opposite vortex and the upwash correction can, however, be separated by use of an iterative scheme that involves the location of the vortex centers and the downwash distribution. An initial estimated value for the influence of the opposite vortex in the pair is found by using the upwash distribution outboard of the vortex center. Removal of the influence of the opposite vortex establishes an isolated upwash profile for each side of the wake; i.e., a wake structure wherein the opposite vortex is assumed to be far away. In the initial step of the iteration a value near zero is assumed for the upwash adjustment or correction. The maximum swirl velocities on the two sides of the vortex center are then compared. If they are not equal, the change in upwash increment needed to make them equal is calculated and iterated upon until they agree within experimental accuracy. After each revised upwash distribution has been found, a new vortex center is located by interpolating along the upwash curve through the core region of the vortex to find the span-
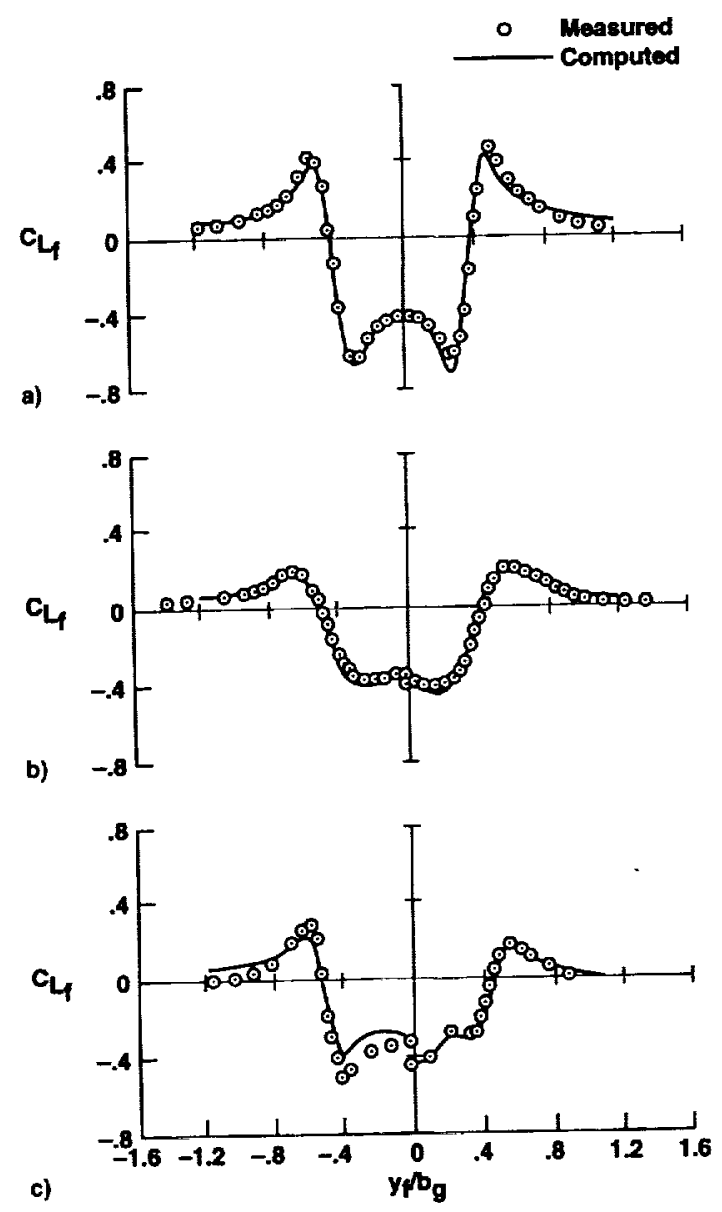

Fig. 8 Comparison of measured lift induced on following wing no. I as it traverses laterally through the wake of various configurations, with that predicted by use of measured downwash and vortex-lattice theory: a) $30 / 30 \mathrm{deg}$, b) $30 / 30-\mathrm{deg}$ fins, and c) $30 / 0 \mathrm{deg}$. 
wise location where the downwash velocity passes through zero. Knowledge of the position of the vortex center makes it possible to determine the swirl velocity as a function of radius for the structure of the opposite vortex so that its contribution can be removed during the next iteration. The entire process converges in about 3-5 iterations.

\section{Comparison of Predicted and Measured Data}

\section{Induced Lift}

The spanwise distributions of up- and downwash angles presented in Fig. 7 were used as input into a vortex-lattice code to predict the lift on following wings nos. 1, 4, and 5 (Figs. 8-10), as they encounter the vortex wake shed by a configuration of the wake-generating model (Table 2). In each part of the figures the computed time-averaged distributions (solid lines) are compared with the measured time-averaged distributions (data shown as open-circle symbols). It is to be noted that the comparisons have been restricted to the timeaveraged data because their use is more straightforward than either the maximum or minimum data sets.

In general, the comparisons in Figs. 8-10 indicate that the lift distributions computed by use of the vortex-lattice method are in good agreement with the measured values. The agreement of the results in Fig. 8 for following model no. 1 are very good considering the complexity and unsteady character of the flowfield. The comparisons made for the larger following models, nos. 4 and 5 , are not as good in the region between the vortex centers. A systematic effort was made to be sure that the discrepancy between the results was not due to misalignments or adjustments in the data nor to approximations
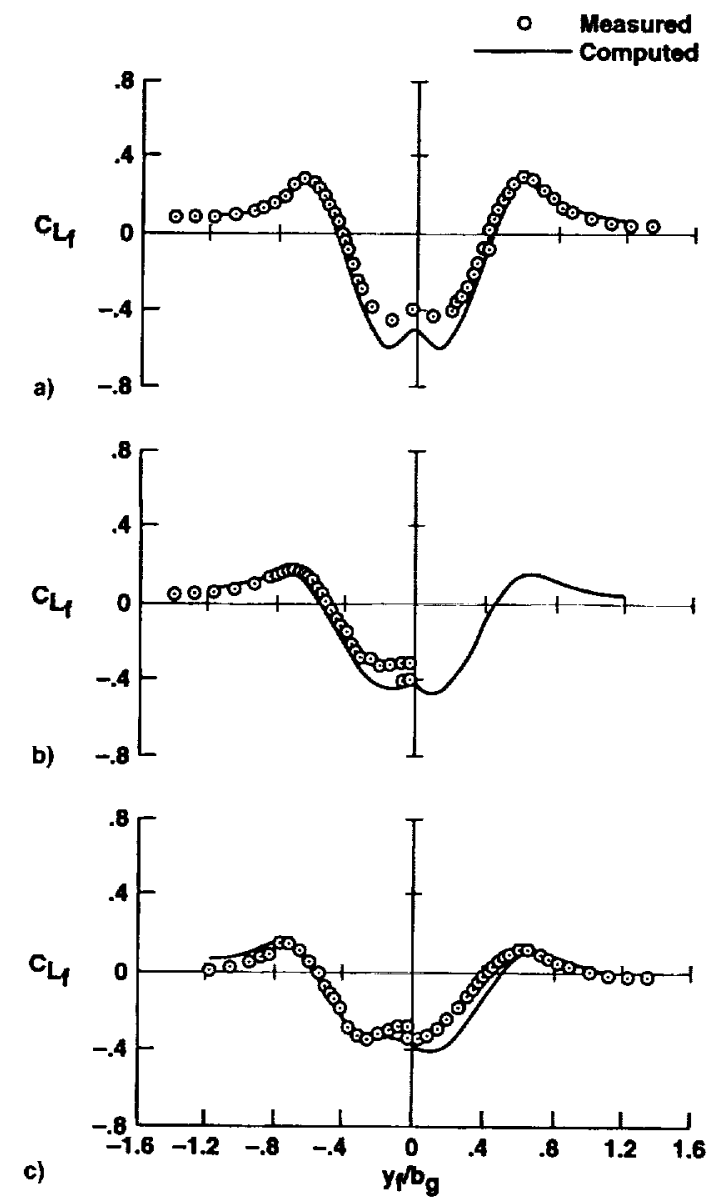

Fig. 9 Comparison of measured lift induced on following wing no. 4 as it traverses laterally through the wake of various configurations, with that predicted by use of measured downwash and vortex-lattice theory: a) 30/30 deg, b) 30/30-deg fins, and c) 30/0 deg.

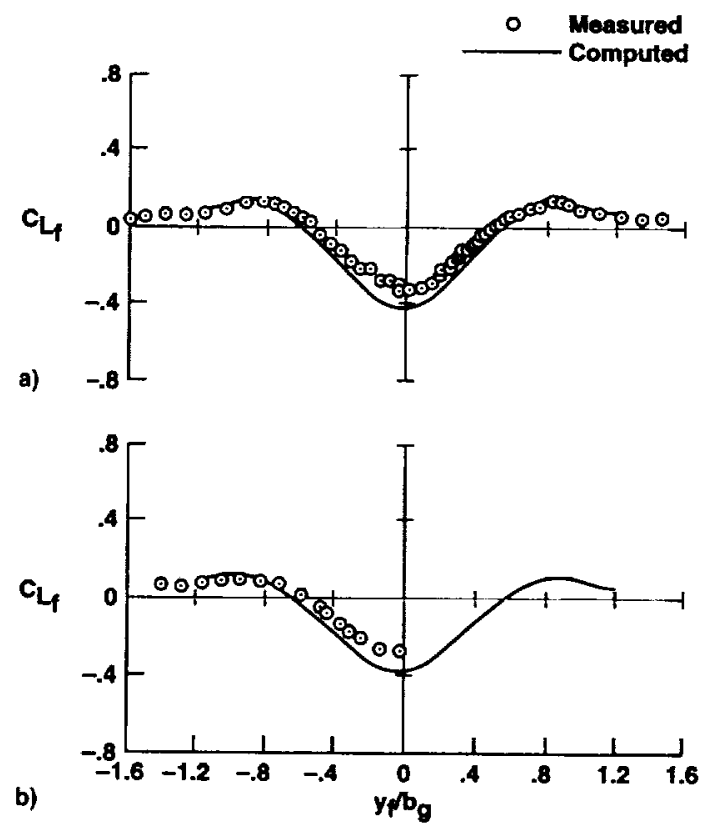

Fig. 10 Comparison of measured lift induced on following wing no. 5 as it traverses laterally through the wake of various configurations, with that predicted by use of measured downwash and vortex-lattice theory: a) $30 / 30 \mathrm{deg}$ and b) 30/30-deg fins.

in the mesh size, spacings, etc., in the vortex-lattice code. Examination of the comparisons for following model no. 1 indicate that the predictions follow the measured data well within experimental accuracy. The larger than usual discrepancy for following model no. 1 in the port vortex behind the $(30 / 0 \mathrm{deg})$ configuration is not unexpected because the alleviation mechanism for that wake did not go to completion for that side of the wake. As a result, two vortices are in close proximity causing the wake to be very unsteady and the rolling moments to be somewhat higher. The generally good agreement in Fig. 8 for following model no. 1 indicates that the assumptions made in order to apply the potential-flow vortexlattice method to the rotational flowfield of vortex wakes are valid approximations. Furthermore, since following model no. 1 is the smallest of the three following wings, it is the one expected to suffer most from flow separation or stall whenever the vortex flowfield impresses a large angle of attack on the following wing. Since no such deviation is noted for following model no. I, flow separation probably is not the cause of the discrepancies that appear for following models nos. 4 and 5 in Figs. 9 and 10, respectively. It is concluded therefore, that the differences between the computed and measured data are due to some characteristic of the flowfield that is not included in the vortex-lattice method or in the application of the boundary conditions. Since the agreement of the predicted and measured data for following wing no. 1 is very good, it is also concluded that the experimental and data reduction techniques are adequate and produce reliable data.

\section{Induced Rolling Moment}

The sequence used for the comparisons presented in Figs. $11-13$ for rolling moment parallel those presented previously for lift. Once again the rolling-moment distributions computed for following model no. 1 (Fig. 11) are in very good agreement with the measured data. The values computed for following model no. 4 (Fig. 12) are also in quite good agreement in most regions except near the vortex centers, where the magnitude of the predictions are again too large. The same comments can be made for the comparisons presented for following model no. 5 (Fig. 13), but the magnitude of the discrepancy has increased over that observed for the two smaller encountering wings. Once again, the discrepancies are be- 


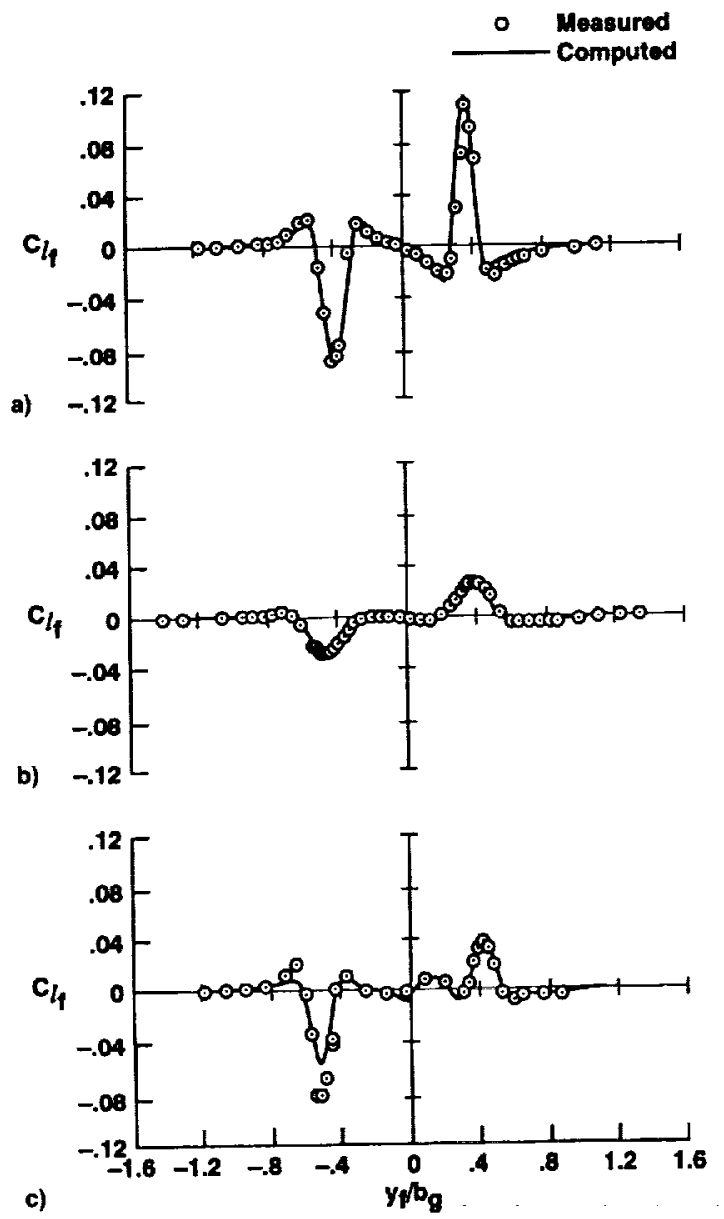

Fig. 11 Comparison of measured rolling moment induced on following wing no. $I$ as it traverses laterally through the wake of various configurations, with that predicted by use of measured downwash and vortex-lattice theory: a) $30 / 30 \mathrm{deg}$, b) 30/30-deg fins, and c) $30 / 0 \mathrm{deg}$.

lieved to be due not to flow separation, nor to lack of accuracy of the application of the vortex-lattice method, but, as discussed in the next section, due to one or more flowfield characteristics not included in the method.

\section{Assessment of Assumptions}

As mentioned previously, a systematic effort was made to be sure that the discrepancy between the results was not due to misalignments or adjustments in the data nor to approximations in the mesh size, spacings, etc., in the vortex-lattice code. It was found that the computed values usually changed less than $1 \%$ at any place along the span due to any of the items tried. After these various possibilities had been tested and eliminated as being responsible for the discrepancy between computed and measured distributions, the assumption made in the application of the vortex-lattice method regarding the streamwise downwash distribution was investigated. That is, when the vertical velocity at each control point is set, it is assumed that the value is constant for all control points at the same spanwise station. In other words, the downwash is assumed to be the same for each set of streamwise panels or lattices and an allowance for distortion of the oncoming vorticity distribution is not made. It is believed that this approximation is the most logical reason for the discrepancy between the computed and measured lift and rolling-moment distributions. In support of this conjecture, it is again noted that the vortex-lattice method was quite accurate when the following wing was small and that the error increased as the following wing increased in size. Similarly, the trend in predictive accuracy follows the fact that small following wings cause small or negligible distortions to a vortex wake; whereas, large wings cause larger distortions in the structure of the vortex wake. Since little distortion is expected when the following wing is small, like following wing no. 1 , the vortexlattice method would be expected to be reliable with only small or negligible error. When the following wing is large, like nos. 4 and 5 , the distortion of the vorticity field and the resulting downwash distribution is expected to be larger, and perhaps even quite significant. It remains to be confirmed that the discrepancies between computed and measured data are caused by vortex-wake distortions brought about by the influence of the following wing. The first requirement is that it be shown that wake distortion is able to bring about the changes in magnitude and distribution observed between the predicted and measured quantities for the larger wings. It would then be desirable to develop an effective method to properly analyze the wing/vortex-wake interaction and apply it to the test configurations studied here.

An estimate of the distortion that can be brought about by a wing/vortex-wake interaction is relatively simple to obtain by use of a Trefftz-plane type of analysis (Fig. 14). The computation was carried out by analyzing the motion of twodimensional point vortices arranged in a circular distribution and with a strength distribution designed to approximate the vorticity distribution typical of lift-generated vortices. The boundary conditions on the motion of the vortices are applied so that, prior to the $x / b_{g}=0$ station, the vortices move as if in two-dimensional free space. Downstream of $x / b_{g}=0$, the vortices above $z / b_{g}=0$ move as if they are over an infinite flat plate, and those below $z / b_{g}=0$ move as if they are under an infinite flat plate. Both of these two motions are simulated

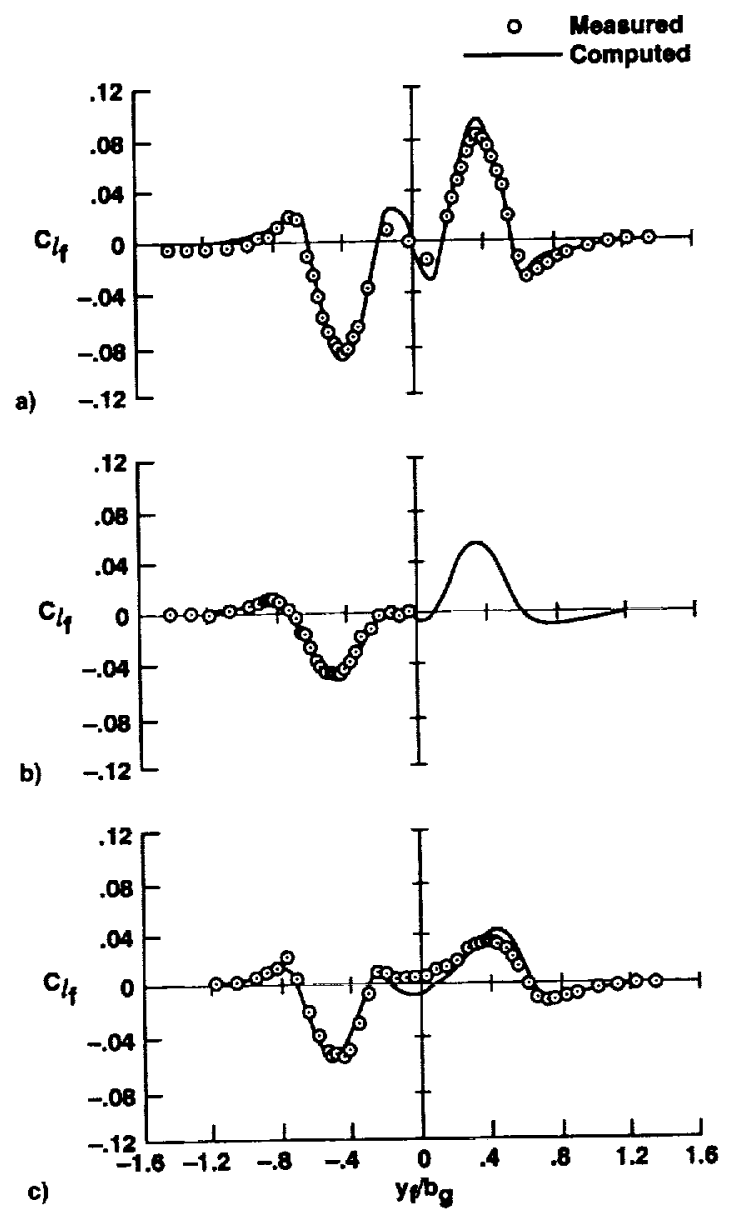

Fig. 12 Comparison of measured rolling moment induced on following wing no. 4 as it traverses laterally through the wake of various configurations, with that predicted by use of measured downwash and vortex-lattice theory: a) $30 / 30 \mathrm{deg}$, b) $30 / 30-\mathrm{deg}$ fins, and c) $30 / 0 \mathrm{deg}$. 

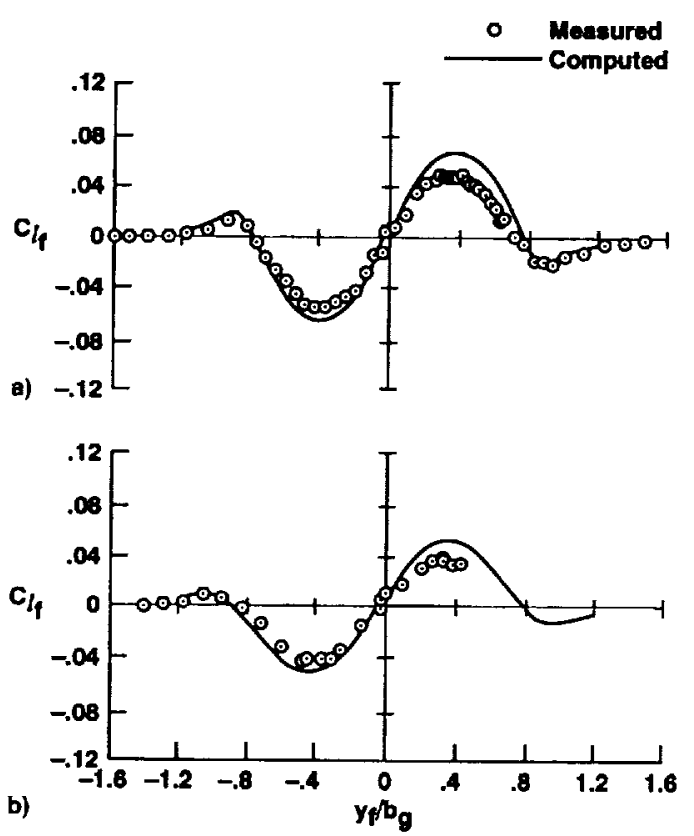

Fig. 13 Comparison of measured rolling moment induced on following wing no. 5 as it traverses laterally through the wake of various configurations, with that predicted by use of measured downwash and vortex-lattice theory: a) 30/30 deg and b) 30/30-deg fins.

in the Trefftz plane by use of images. At each of the $x / b_{g}$ stations specified in Fig. 14, the infinite plate is assumed to end. The downwash distribution is then calculated for the position of the vortices at that station as if the plate (and the images) were not there. The various parts of Fig. 14 represent the amount of interaction for following wings of different sizes as indicated by the $x / b_{g}$ values noted in each part of Fig. 14. That is, the upper figure for $x / b_{s}=0$ represents the downwash induced in the freestream by the undisturbed vortex wake. Each figure that follows illustrates the distortion in the wake caused by a following wing of larger size and the downwash induced by the new wake shape.

The examples presented in Fig. 14 assumed that the leading edge of the flat plate was sharp and thin enough to divide the vortex in half as it encounters the wing. Therefore, the calculations presented in Fig. 14 do not simulate the bluntness of the leading edge of the following wings. Since the small following wings are thin compared with the depth of the wake, a zero thickness is a valid approximation. However, when the following wing is large, the bluntness of the leading edge and the thickness of the wing might be enough to cause the highspeed vortex core to stay intact and to pass either above or below the wing rather than being split in half as assumed in Fig. 14. If this occurs, sample computations indicate that the resulting downwash distributions are as a whole about the same as the thin-wing results, but the distributions differ in detail.

It is noted in Fig. 14 that those point vortices on top of the flat plate or wing move outboard with time, and those under the flat plate move inboard with time, just as anticipated intuitively. This movement redistributes the vorticity in the wake so that the downwash in the spanwise region between the two vortex centers is reduced, just as observed with the lift for the larger following wings. Furthermore, the outboard distributions of downwash are slow to be altered, just as observed with the lift comparisons. Also, it is noted that the total change in downwash velocity across the vortex centers is slow to be altered so that the rolling moment on an encountering wing would also be slow to be changed by the wake distortion. The computation was also carried out for vortex distributions that approximate solid-body rotation, and the results were about the same, but took longer to occur.
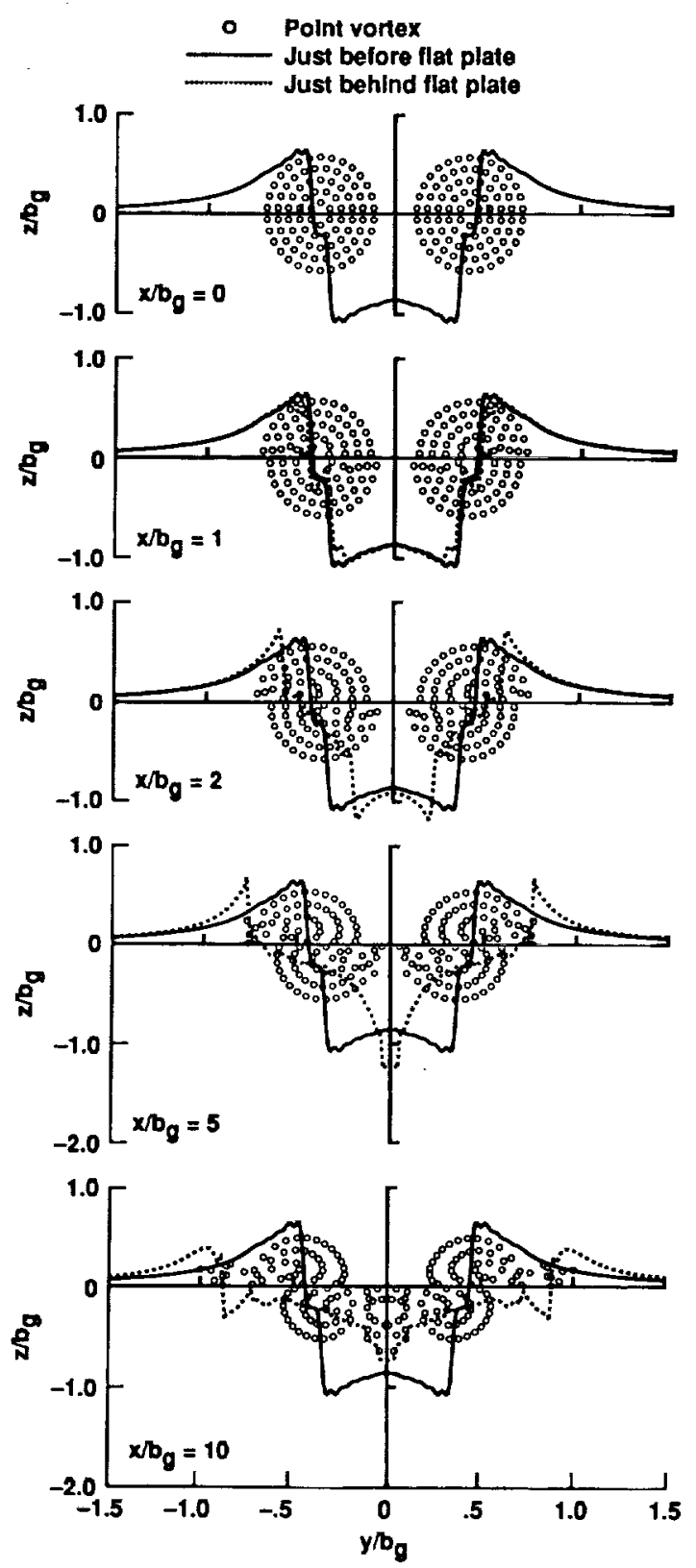

Fig. 14 Distortion of vorticity distribution in a vortex wake as it encounters a flat plate of various downstream sizes.

Not in agreement with the experimental data is the time or streamwise distance required for the chord of the following wing to accomplish the vorticity distortions indicated in Fig. 14. It is believed that the Trefftz-plane approximation does not properly represent the continuation of the vorticity redistribution as the wake passes downstream of the trailing edge of the following wing, nor as it interacts with the vortex wake of the following wing. Even without inclusion of this effect, the qualitative aspects of the changes in downwash distribution indicate that the ingredient left out of the current vortex-lattice method is the distortion of the vorticity distribution in the vortex wake that is brought about by the presence of the encountering wing. The experimental method and the adjustments to the data continue to appear to be viable techniques.

\section{Concluding Remarks}

Results are reported here on a study of the reliability of a vortex-lattice method for the computation of lift and rolling moment induced on following wings by an oncoming stream that contains the vortex wake of a subsonic transport aircraft 
configured for landing. The vortex-lattice method is tested by using it as the link between the measured velocity distribution and measured induced loads in the same vortex wake. The downwash, lift, and rolling-moment distributions used in the present study were all obtained during a test program carried out previously in the 80 by $120 \mathrm{ft}$ Wind Tunnel.

Comparison of the vortex-lattice predictions with the corresponding values obtained in the wind tunnel indicates that the method is reliable for small following wings. However, as the span and planform area of the encountering wing become larger, it was found that the differences between the two results becomes larger. The magnitudes of the discrepancies between the computed and the measured values are much larger than those observed between different ground-based facilities or between tests in the same facility. The effect of mesh size and spacing in the vortex-lattice method and various data adjustments on the vortex-lattice results indicate that some other feature of the method was responsible for the lack of agreement when large following wings are studied. After further study, it was concluded that the rotational flowfield of the vortex wake is altered enough by the encountering wing to account for the discrepancy between the vortex-lattice and measured results. It was also concluded that the experimental technique was reliable and not responsible for the observed discrepancies.

\section{References}

'Hough, G., "Remarks on Vortex-Lattice Methods," Journal of Aircraft, Vol. 10, No. 5, 1973. pp. 314-317.

=Rossow, V. J., Corsiglia, V. R., Schwind, R. G., Frick, J. K. D., and Lemmer, O. J.. "Velocity and Rolling-Moment Measurements in the Wake of a Swept-Wing Model in the 40- by 80-Foot Wind Tunnel," NASA TM X-62,414, April 1975.
${ }^{3}$ Rossow, V. J., Sacco, J. N., Askins, P. A., Bisbee, L. S., and Smith, S. M., "Measurements in 80- by 120-Foot Wind Tunnel of Hazard Posed by Lift-Generated Wakes," Journal of Aircraft, Vol. 32. No. 2, 1995, pp. 278-284; also AIAA Paper 93-3518, Aug. 1993.

'Holbrook, G. T., "Vortex Wake Hazard Analysis Including the Effect of the Encountering Wing on the Vortex," M.S. Thesis, George Washington Univ., Washington, DC, Aug. 1985.

'Stewart, E. C., "A Study of the Interaction Between a Wake Vortex and an Encountering Airplane," AIAA Paper 93-3642, Aug. 1993.

"Jones, W. P., "Vortex-Elliptic Wing Interaction," AlA A Journal,

Vol. 10, No. 2, 1972, pp. 225-227.

'Filotas, L. T., "Vortex Induced Wing Loads," AIAA Journal, Vol. 10, No. 7, 1972, p. 971

'Barrows, T. M., "Simplified Methods of Predicting Aircraft Rolling Moments Due to Vortex Encounters," Journal of Aircraft, Vol. 14. No. 5, 1977, pp. 434-439.

"Flax, A. H., "Comment on 'Simplified Methods of Predicting Aircraft Rolling Moments Due to Vortex Encounters,'" Journal of Aircraft, Vol. 14, No. 11, 1977, pp. 1151, 1152.

"'Barrows, T. M., "Reply to A. H. Flax," Journal of Aircraft, Vol. 14, No. 11, 1977, p. 1152.

"Kellogg. O. D., Foundations of Potential Theory, Dover, New York, 1953, pp. 160-176.

"Heaslet, M. A., and Spreiter, J. R., "Reciprocity Relations in Acrodynamics," NACA Rept. 1119, 1953.

${ }^{13}$ Munk, M. M., Fundamentals of Fluid Dynamics for Aircraft Designers, Ronald, New York, 1929.

"Rossow, V. J., "Estimate of Loads During Wing-Vortex Interactions by Munks Transverse-Flow Method," Journal of Aircraft, Vol. 27, No. 1, 1990, pp. 66-74.

${ }^{15}$ Rossow, V. J., "Effect of Wing Fins on Lift-Generated Wakes," Journal of Aircraft. Vol. 15, No. 3, 1978, pp. 160-167.

In Rossow, V. J., "Experimental Investigation of Wing Fin Configurations for Alleviation of Vortex Wakes of Aircraft," NASA TM 78520, Nov. 1978. 
